\title{
Tibio-fibular synostosis
}

INSERM

\section{Source}

INSERM. (1999). Orphanet: an online rare disease and orphan drug data base. Tibio-

fibular synostosis. ORPHA:295028

Tibio-fibular synostosis is a rare, non-syndromic limb malformation characterized by fusion of the proximal or distal tibial and fibular metaphysis and/or diaphysis, frequently associated with distal positioning of the proximal tibiofibular joint, leg length discrepancy, bowing of the fibula, and valgus deformity of the knee. 